\title{
The Evolution of Strain Gradient and Anisotropy in Gradient-Structured Metal
}

\begin{abstract}
XIANGDE BIAN, FUPING YUAN, XIAOLEI WU, and YUNTIAN ZHU
Gradient-structured metals have been reported to possess superior mechanical properties, which were attributed to their mechanical heterogeneity. Here we report in-situ observation of the evolution of strain gradient and anisotropy during tensile testing of a gradient-structured metal. Strain gradients and anisotropy in the lateral directions were observed to increase with increasing applied tensile strain. In addition, the equivalent Poisson's ratio showed gradient, which evolved with applied strain. The gradient structure produced higher deformation anisotropy than coarse-grained homogeneous structure, and the anisotropy increased with increasing tensile strain. The strain gradient and anisotropy resulted in strong back-stress hardening, large strain gradients, and a high density of geometrically necessary dislocations, which helped with increasing the ductility.
\end{abstract}

DOI: $10.1007 / \mathrm{s} 11661-017-4199-2$

(C) The Minerals, Metals \& Materials Society and ASM International 2017

\section{INTRODUCTION}

IT has been a challenge to produce both high strength and high ductility in metals and alloys. ${ }^{[1-5]}$ The strength can be easily enhanced by well-known strategies such as grain refinement, work hardening, solution strengthening, second-phase particle strengthening, and deformation twins. ${ }^{[6-11]}$ However, high strength is usually accompanied with the sacrifice of ductility in homogeneous metals. For example, ultrafine-grained (UFG) or nanostructured (NS) metals obtained by severe plastic deformation (SPD) can have strengths an order of magnitude higher than those of their coarse-grained (CG) counterparts. However, they usually show limited strain hardening and very low (near zero) uniform elongation. ${ }^{[6,7,12,13]}$

Recently, several promising strategies for achieving simultaneous high strength and high ductility have been proposed by tailoring microstructures through heterogeneous and/or hierarchical structures. ${ }^{[2-5,14-29]}$ Among them, the gradient structure, where the grain size or

XIANGDE BIAN, FUPING YUAN, and XIAOLEI WU are with the State Key Laboratory of Nonlinear Mechanics, Institute of Mechanics, Chinese Academy of Sciences, Beijing 100190, P.R. China, and also with the School of Engineering Science, University of Chinese Academy of Sciences, Beijing 100190, P.R. China. Contact e-mail: fpyuan@Inm.imech.ac.cn YUNTIAN ZHU is with the Nano Structural Materials Center, School of Materials Science and Engineering, Nanjing University of Science and Technology, Nanjing 210094 , Jiangsu, P.R. China, and also with the Department of Materials Science and Engineering, North Carolina State University, Raleigh, NC 27695.

Manuscript submitted March 19, 2017.

Article published online July 10, 2017 the substructure size changes gradually along the depth, ${ }^{[5,20-26]}$ has great potential in engineering applications due to their superior combinations of strength and ductility. The tensile properties and underlying deformation mechanisms of the gradient-structured metals have been reported recently. ${ }^{[5,20,21,23-26]}$ For example, gradient-structured $\mathrm{Cu}$ was reported 100 pet stronger than $\mathrm{CG} \mathrm{Cu}$, while retaining its ductility, ${ }^{[20]}$ which was attributed to the growth of nanocrystalline layer due to the low thermal stability of $\mathrm{Cu}$. In gradient-structured metals with stable microstructures, the high ductility was attributed to the presence of strain gradient together with stress-state change, which promotes dislocation interactions and generation of geometrically necessary dislocations (GNDs). ${ }^{[21,24,30]}$

In addition to forest dislocation hardening, backstress strengthening and back-stress work hardening have also been reported to play an important role in the deformation of heterogeneous structures, such as the gradient structure and heterogeneous lamella structure. ${ }^{[19,24,28]}$ For the gradient structure, the onset of plastic deformation occurs in soft CG core first, and then propagate gradually to the hard surface layer with increasing applied tensile strain. ${ }^{[5,20]}$ This progressive yielding of gradient layers results in gradient plastic strain in the gradient-structured samples, as revealed recently by a crystal-plasticity finite element modeling. ${ }^{[25]}$ The plastic strain gradient needs to be accommodated by GNDs, which in turn produces significant back stress. ${ }^{[19,24,28,31-35]}$ This can simultaneously increase strength as well as ductility.

The gradient structures can be regarded as consisting of numerous thin layers with systematically varying mechanical behaviors. Therefore, mechanical 
incompatibilities exist between neighboring layers during tensile testing, ${ }^{[5,21,24,25]}$ which produces strain gradient and enhanced mechanical properties. However, how the strain gradient and anisotropy are generated and developed during tensile loading is poorly understood, because of the lack of experimental study. It is of critical importance to investigate the evolution of strain gradient and anisotropy in gradient-structured materials to better understand the scientific principles behind their observed unique tensile behavior and superior mechanical properties.

In this study, quasi-static tensile testing was coupled with in-situ 3D strain mapping on lateral sample surfaces using digital image correlation (DIC) to investigate the evolution of strain gradient and anisotropy. Loading-unloading-reloading (LUR) tests were conducted to study the back-stress evolution. It was revealed for the first time that strain gradient and anisotropy increased with increasing applied strain, indicating back-stress work hardening associated with increasing GND density.

\section{MATERIALS AND EXPERIMENTAL PROCEDURES}

\section{A. Materials}

A TWIP steel was used in this work to study the evolution of strain gradient and anisotropy in the gradient-structured sample under tensile load. The steel has a chemical composition of $0.6 \mathrm{C}, 23 \mathrm{Mn}, 0.035 \mathrm{Nb}$, $0.035 \mathrm{Ti}$, and the balance of Fe (all in wt percent). The initial material was first melted in an induction furnace under the protection of Ar atmosphere and then cast into an ingot with dimensions of $750 \times 270 \times 170 \mathrm{~mm}$. The ingot was homogenized at $1423 \mathrm{~K}\left(1150{ }^{\circ} \mathrm{C}\right)$ for 2 hours, and then hot-forged between $1423 \mathrm{~K}$ and $1173 \mathrm{~K}\left(1150{ }^{\circ} \mathrm{C}\right.$ and $\left.900{ }^{\circ} \mathrm{C}\right)$ to form slabs with a thickness of $20 \mathrm{~mm}$. Gradient structures were produced by surface mechanical attrition treatment (SMAT). The disks for SMAT were cut from the slab by wire saw into dimensions of $70 \times 50 \times 3 \mathrm{~mm}$. These disks were fully austenized at $973 \mathrm{~K}\left(700{ }^{\circ} \mathrm{C}\right)$ for 1 hour followed by immediate quenching in water. This produced samples with a $\mathrm{CG}$ microstructure with an average grain size of about $15 \mu \mathrm{m}$ (Figure 1(d)). Both sides of the sample plates were SMAT-processed by high-velocity steel balls (4 $\mathrm{mm}$ in diameter) for 30 minutes. More information on SMAT can be found elsewhere. ${ }^{[36]}$

\section{B. Microstructural Characterization}

Back-scattered electron (BSE) imaging, electron back-scattered diffraction (EBSD), and transmission electron microscopy (TEM) were used to study the microstructures and their gradients. The samples for BSE and EBSD were first grinded to 2000 grit with sandpapers, and then polished with a $0.05 \mu \mathrm{m} \mathrm{SiO}_{2}$ aqueous suspension, followed by electro-polishing in a solution of 10 pet $\mathrm{HCLO}_{4}$ and 90 pet alcohol at $22 \mathrm{~V}$ and $253 \mathrm{~K}\left(-20^{\circ} \mathrm{C}\right)$. Thin disks with thickness of about
$300 \mu \mathrm{m}$ were prepared for TEM observations, and then mechanically ground to about $50 \mu \mathrm{m}$, followed by a twin-jet polishing using a solution of 5 pet perchloric acid and 95 pct ethanol at $22 \mathrm{~V}$ and $253 \mathrm{~K}\left(-20^{\circ} \mathrm{C}\right)$. $\mathrm{X}$-ray diffraction (XRD) measurements were performed on polished samples to obtain the phase and texture information using a Philips Xpert X-ray diffractometer with $\mathrm{Cu} \mathrm{K} \mathrm{K}_{\alpha}$ radiation. The distribution of micro-hardness along the sample depth was obtained on the polished cross section using a Vickers diamond indenter under a load of $10 \mathrm{~g}$ for 15 -second dwell time. For each depth, five groups of measurements were made.

\section{Quasi-Static Uniaxial Tensile Tests Coupled with Digital Image Correlation}

Dog-bone samples, with a thickness of about $3 \mathrm{~mm}$, a gage length of $15 \mathrm{~mm}$, and a width of $4 \mathrm{~mm}$, were used for quasi-static tensile tests and LUR tests. These tensile tests and LUR tests were conducted at a strain rate of $5 \times 10^{-4} / \mathrm{s}$ and at room temperature under displacement control using an Instron 5565 testing machine. The tensile, thickness, and width directions are designated as the $y, z$, and $x$ directions, respectively, as shown in Figure 2(c). Strain contours were measured using digital image correlation (DIC) during tensile tests. A commercial software, ARAMIS ${ }^{\circledR}$, was used to analyze the DIC data. Initial high-contrast stochastic spot patterns on both the treated surface ( $x y$ plane) and the lateral surface ( $y z$ plane) were created, in which a background of flexible, adhesive, and matte white paint was sprayed first, and then a fine layer of black paint spots (with size of about $10 \mu \mathrm{m}$ ) was sprayed onto the white background. The evolution of the spot patterns was recorded using two 1.2 MPx digital CCD cameras at a rate of 1 frame per second to calculate the contours for the in-plane strain and the out-plane height profile for both the treated surface and the lateral surface. The facet size for the strain calculation using DIC method was $50 \mu \mathrm{m}$.

\section{EXPERIMENTAL RESULTS AND DISCUSSIONS}

\section{A. Microstructural Characterization and Tensile Properties}

Figure 1 shows the microstructures for the gradient structure after SMAT. Figure 1(a) shows the BSE image for the whole gradient structure. The close-up BSE image of the treated surface is displayed in Figure 1(b). Figure 1(c) shows the TEM image at the depth of $50 \mu \mathrm{m}$ from the treated surface. The EBSD (IPF) images, textures, and intensities at various depths for the gradient structure are shown in Figures 1(e1) through (e4) and (f1) through (f4), respectively. It is observed that the grain itself is not refined and the grain size is similar at various depths (about $15 \mu \mathrm{m}$ ), while substantial substructures with much refined size in the interior of grains are formed on the treated surface. These substructures are identified as single deformation twins or multiple deformation twins from the TEM image 

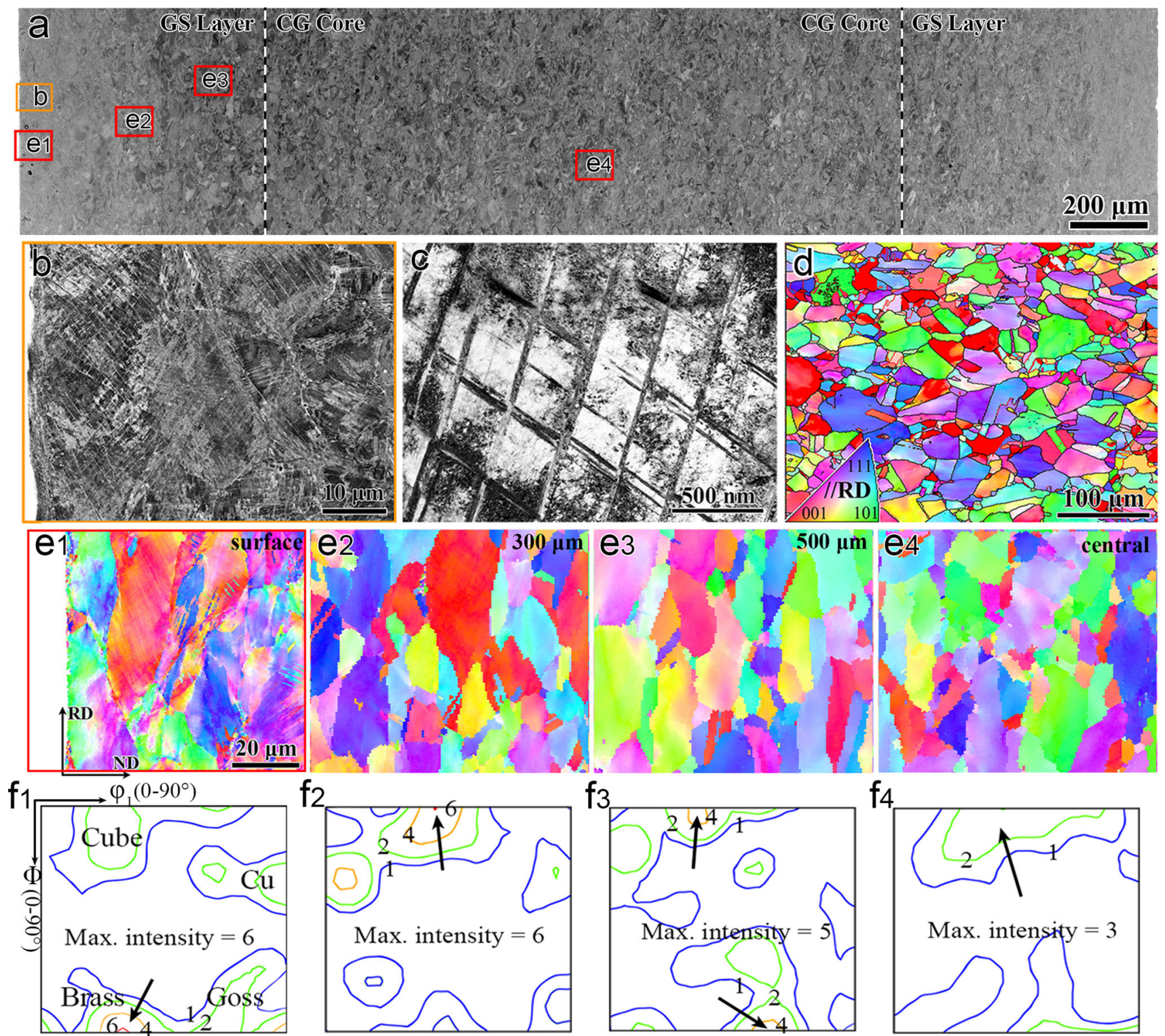

$\mathrm{f}_{4}$

Fig. 1-(a) Back-scattered electron (BSE) micrograph for the whole gradient structure; $(b)$ BSE micrograph at the treated surface; $(c)$ TEM image at the depth of $50 \mu \mathrm{m}$ from the treated surface; (d) EBSD (IPF) image for the untreated CG sample; (e) EBSD (IPF) images at various depths for the gradient structure: (e1) surface, $(e 2) 300 \mu \mathrm{m},(e 3) 500 \mu \mathrm{m},(e 4)$ center; $(f)$ Texture and intensity at various depths for the gradient structure: (f1) surface, (f2) $300 \mu \mathrm{m},(f 3) 500 \mu \mathrm{m}$, (f4) center.

(Figure 1(c) clearly shows multiple twins from two twinning systems). Twin boundary spacing is less than $300 \mathrm{~nm}$. High density of dislocations can also be found in the areas near twin boundaries. Such microstructure refinement by deformation twins and dislocation tangles result in the reduction of dislocation mean free path in the grain interior, rendering an obvious hardness increase at the treated surface. Previous research ${ }^{[16]}$ also revealed that hierarchical deformation twins can produce higher strength/hardness than a single level of primary deformation twins. Moreover, the gradient structure shows weak texture, as indicated from the texture intensity maps (the maximum intensity is less than 6) at various depths in Figures 1(e1) through (e4). Thus, the texture effect on the deformation anisotropy is not considered in the present work.
Figure 2(a) shows the distribution of micro-hardness along the depth for the gradient structure after SMAT for 30 minutes. The $1.5-\mathrm{mm} \mathrm{CG}$ core is sandwiched between two $0.6-\mathrm{mm}$ gradient layers. As shown, the micro-hardness decreases almost linearly from about $550 \mathrm{Hv}$ at the surface to about $345 \mathrm{Hv}$ at the center. This micro-hardness distribution along the depth is caused by the gradient twinned structure. Figure 2(b) displays the XRD curves from the untreated CG sample and the SMAT-processed sample surface, which clearly indicates that there is no phase transformation during the SMAT process. The engineering stress-strain curves for the untreated CG sample and the gradient-structured sample are displayed in Figure 2(d). The CG sample has a yield strength of about $400 \mathrm{MPa}$ and a uniform elongation of about 65 pct. As shown, the 


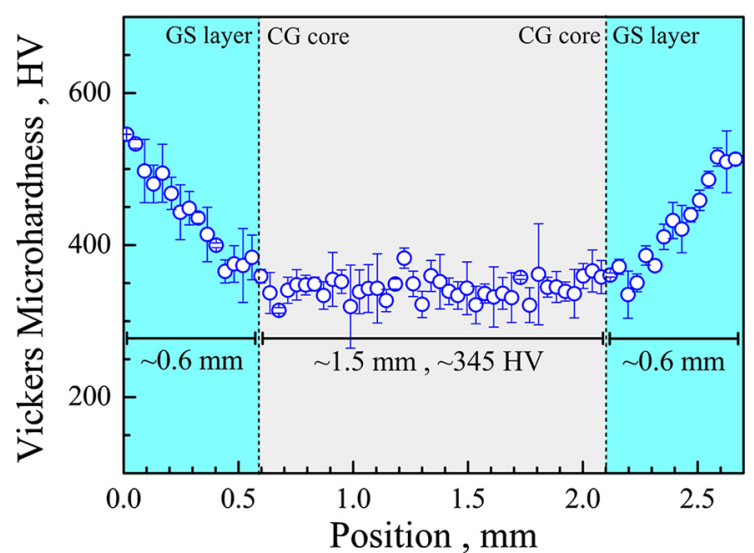

(a)

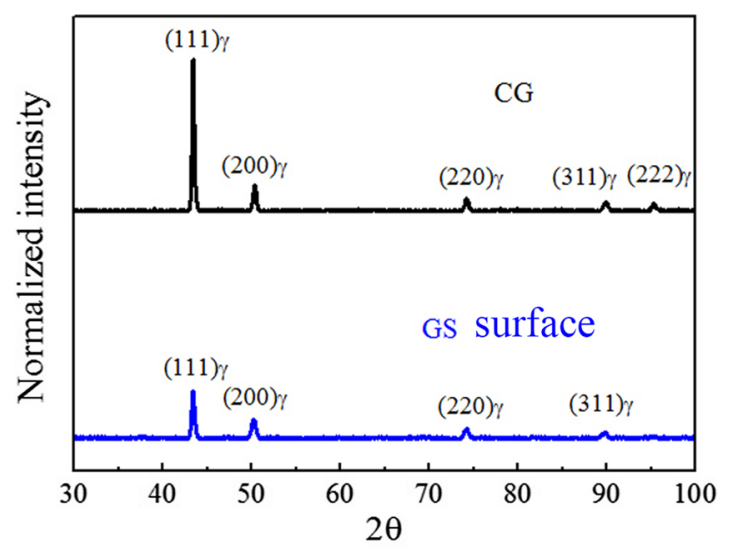

(b)

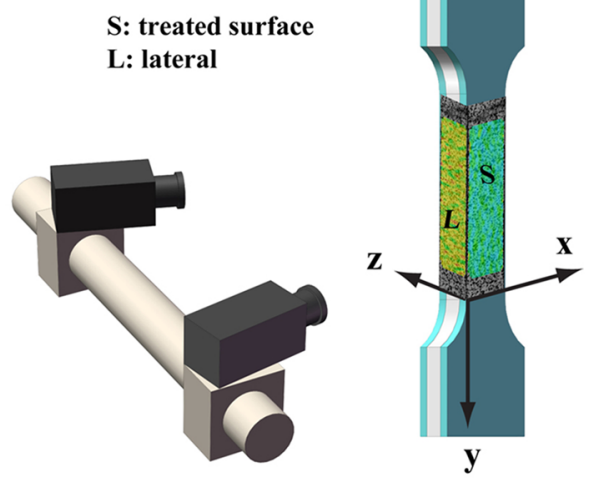

(c)

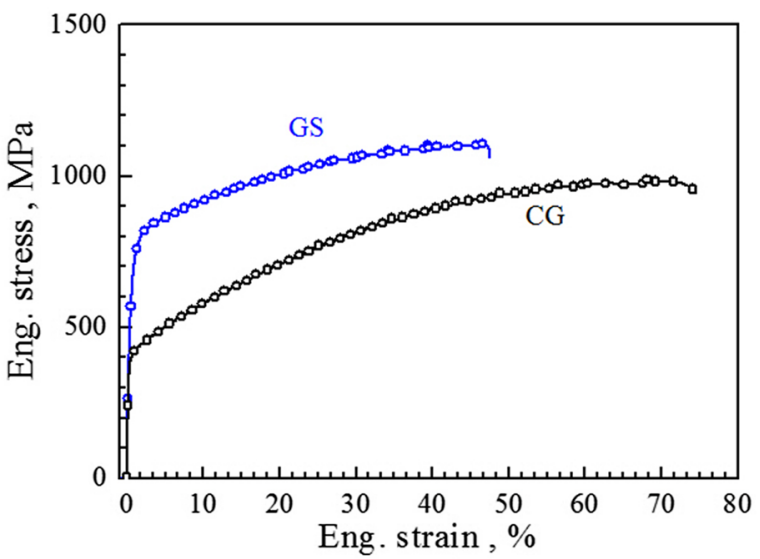

(d)

Fig. 2-(a) Vickers micro-hardness distribution along the depth for the gradient structure; $(b)$ XRD curves for the untreated CG sample and the surface after SMAT; $(c)$ Illustration of experimental set-up for the tensile tests coupled with DIC; $(d)$ Engineering stress-strain curves for the CG and the gradient structure.

gradient-structured sample achieved a superior combination of strength and ductility: its yield strength is about two times that of the CG sample and its uniform elongation still retained about 70 pet of that of the $\mathrm{CG}$ sample. Our previous paper ${ }^{[21]}$ attributed these outstanding tensile properties to the extra strain hardening by the strain gradient and the stress-state change due to the mechanical incompatibility. In order to provide better understanding for the detailed mechanisms underlying the observed tensile properties for the gradient-structured sample, how the strain heterogeneity and gradient are generated and developed in the gradient structure will be delineated in the following sections.

\section{B. Strain Contours and Strain Distributions Along the Depth}

As mentioned earlier, the in-plane strain contours and the contours for the out-plane height profile for both the treated surface and the lateral surface were measured by the $3 \mathrm{D}$ DIC techniques. The distribution of strain $\varepsilon_{x}$ on the lateral surface was calculated using the following equation: $\varepsilon_{x}=\varepsilon_{x}^{\text {surface }}+2 \frac{\Delta H}{b_{0}}$, where $\varepsilon_{x}^{\text {surface }}$ is the average strain along the $x$ direction measured from the treated surface, $\Delta H$ is the height difference (in $x$ direction) between each depth and the surface layer measured from the lateral surface, and $b_{0}$ is the width of the samples.

Strain measurements for the untreated CG sample are shown in Figure 3. Contours of $\varepsilon_{z}$ and contours of height profiles for the lateral surface at various tensile strains are displayed in Figures 3(a) and (b), respectively. As indicated from the contours, the deformation was not uniform microscopically even in the homogenous CG structure; this is due to the variation in grain size and orientation in the CG sample. Taking the average value along the $y$ direction for the contours in Figure 3(a), $\varepsilon_{z}$ distributions along the depth at varying tensile strains are shown in Figure 3(c). Similarly, height profile distributions along the depth at various tensile strains can be obtained based on the contours in Figure 3(b), and are displayed in Figure 3(d). Then the $\varepsilon_{x}$ distributions along the depth at varying tensile strains can be calculated by the aforementioned equation and are shown in Figure 3(e). Although microscopic heterogeneity is observed in the strain contours, relatively homogeneous deformation along the depth is observed for the CG structure when taking the average value 


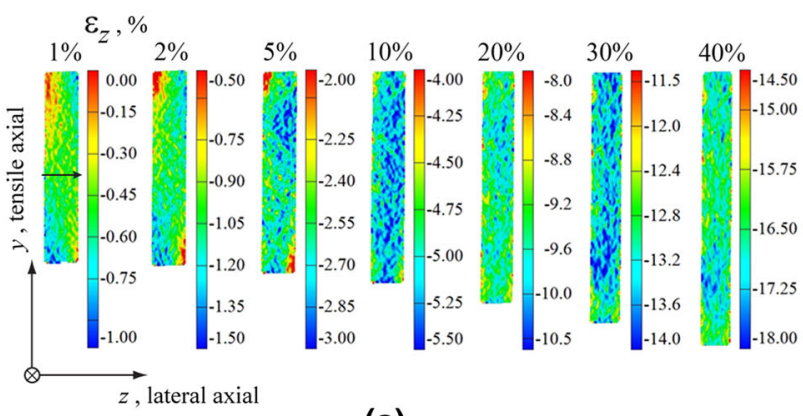

(a)

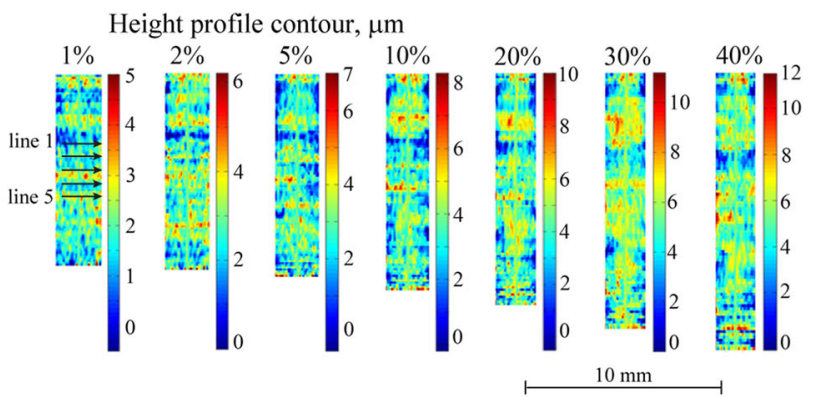

(b)

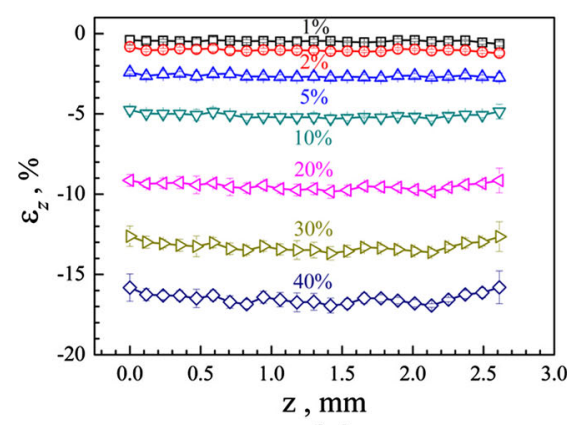

(c)

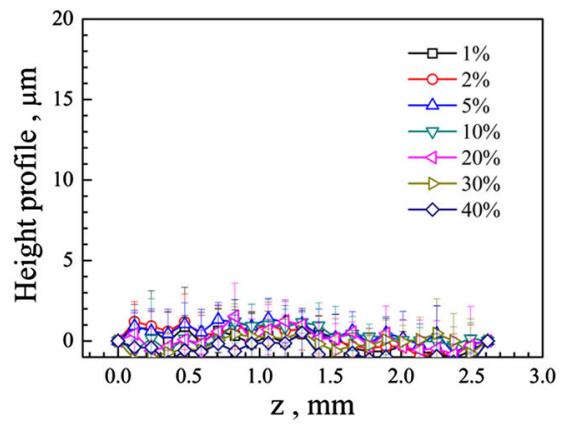

(d)

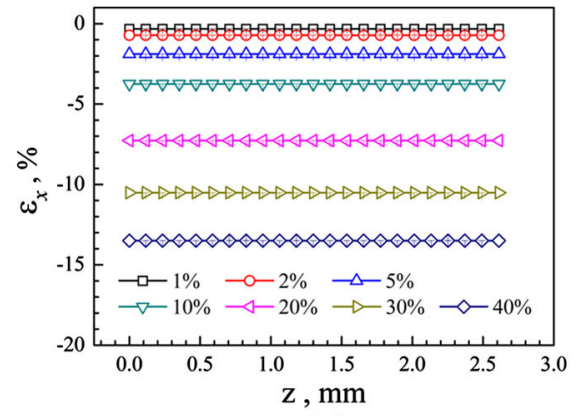

(e)

Fig. 3-Strain measurements for the homogeneous CG structure. (a) Contours of $\varepsilon_{z}$ for the lateral surface at various tensile strains; $(b)$ Contours of height profiles for the lateral surface at various tensile strains; $(c) \varepsilon_{z}$ distributions along the depth at various tensile strains; $(d)$ Height profile distributions along the depth at various tensile strains; (e) $\varepsilon_{x}$ distributions along the depth at various tensile strains.

along the $y$ direction for each layer. As indicated by Figures 3(c) and (e), the lateral deformations $\left(\varepsilon_{x}\right.$ and $\left.\varepsilon_{z}\right)$ are nearly homogeneous along the depth for the untreated CG sample, and this means that no deformation incompatibility and mechanical interactions exist among different layers at various depths for the homogeneous CG structure.

Figure 4 presents the evolution of strains in the gradient-structured sample using the same analysis procedure as for the Figure 3. Again, Figures 4(a) and (b) show the contours of $\varepsilon_{z}$ and the height profiles of the lateral surface at various tensile strains, respectively. Figures 4(c) through (e) display $\varepsilon_{z}$, height profile, and $\varepsilon_{x}$ distributions along the thickness at various tensile strains. As shown in Figures 4(c) and (e), both $\varepsilon_{x}$ and $\varepsilon_{z}$ have gradients along the depth. The strain in the width direction, $\varepsilon_{x}$, is higher at the treated surface than the center part, while the strain in the thickness direction, $\varepsilon_{z}$, shows the opposite trend.

\section{The Evolution of Strain Gradient and Anisotropy}

The evolution of differences in lateral strains at the various depths from that at the central part, $\Delta \varepsilon_{x}$ and $\Delta \varepsilon_{z}$, are shown in Figures 5(a) and (b), respectively. Figures 5(c) and (d) show the normalized lateral strains $\left(\varepsilon_{x} / \varepsilon_{y}\right.$ and $\left.\varepsilon_{z} / \varepsilon_{y}\right)$ as a function of the applied tensile strain at various depths for the gradient-structured samples. The corresponding curves for the untreated CG sample are also included in Figures 5(c) and (d) for comparison. As indicated in Figures 5(c) and (d), the lateral strain $\varepsilon_{x}$ at various depths for the gradient-structured sample is always smaller than that for the untreated CG sample, while the lateral strain $\varepsilon_{z}$ at various depths for the gradient structure is always larger than that for the untreated CG sample. These results indicate that the gradient structure would cause higher anisotropy at the two lateral directions than the homogeneous CG structure. This phenomenon will be discussed further later.

Figure 5 reveals that strain gradient starts at the beginning of the tensile deformation in the gradient-structured sample. The mechanical incompatibilities along the lateral directions should cause constraints and interactions among different layers, and this will lead to lateral stresses in both lateral directions, resulting in a stress-state change from the uniaxial stress state to the multiaxial stress state. ${ }^{[21]}$ As shown in Figures 5(a) and (b), the absolute magnitudes of the strain gradients along the depth $\Delta \varepsilon_{x}$ and $\Delta \varepsilon_{z}$ increase with increasing applied tensile strain. This means that the constraints and interactions between different layers became stronger and stronger with tensile loading. These observations can be attributed to the gradient microstructures and the corresponding gradient mechanical properties along the depth. These strain gradients are in contrast to the well-known strain-gradient plasticity induced by imposing a non-uniform deformation such as bending, ${ }^{[37]}$ torsion, ${ }^{[38]}$ and indentation. ${ }^{[39]}$ However, both situations can produce long-range strain gradients, which are accommodated by the GNDs. ${ }^{[40]}$

For materials with anisotropic properties, the strains for two lateral directions are generally different ( $y$ is the tensile direction): $\varepsilon_{x} \neq \varepsilon_{z}$. It should be noted that the $\varepsilon_{x}$, $\varepsilon_{y}$, and $\varepsilon_{z}$ in this paper are all true strains. In order to 


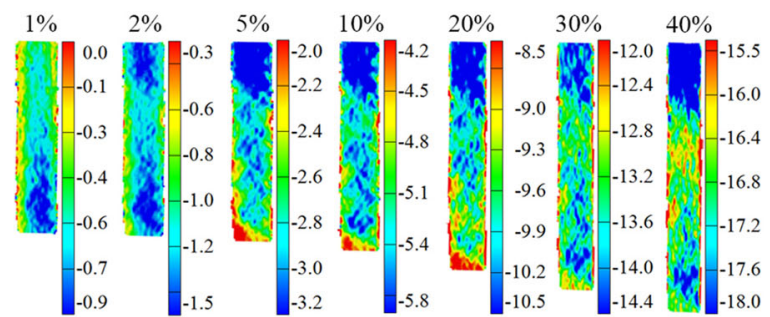

(a)

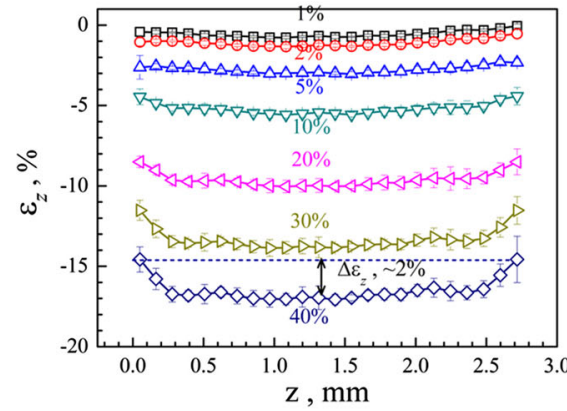

(c)
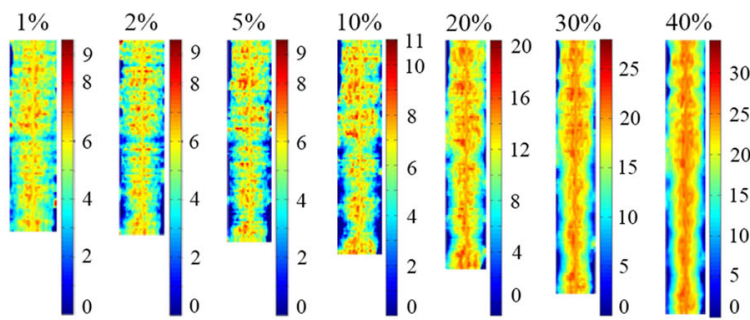

(b)

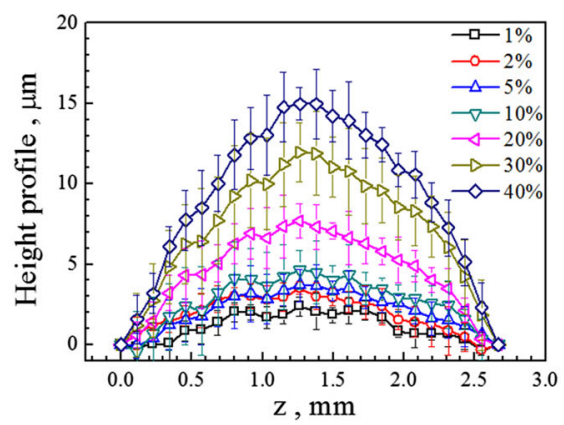

(d)

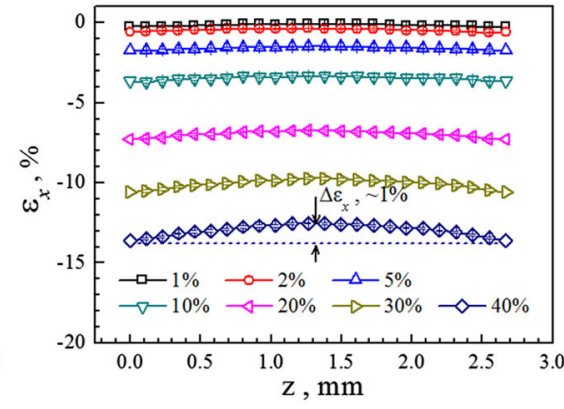

(e)

Fig. 4-Strain measurements for the gradient structure. (a) Contours of $\varepsilon_{z}$ for the lateral surface at various tensile strains; (b) Contours of height profiles for the lateral surface at various tensile strains; $(c) \varepsilon_{z}$ distributions along the depth at various tensile strains; $(d)$ Height profile distributions along the depth at various tensile strains; $(e) \varepsilon_{x}$ distributions along the depth at various tensile strains.

compare the lateral strains of the gradient-structured sample with those of isotropic materials, an equivalent lateral strain and an equivalent apparent Poisson's ratio are defined as following for anisotropic materials:

$$
\begin{gathered}
\bar{\varepsilon}_{\text {lateral }}=\ln \frac{\sqrt{A}}{\sqrt{A_{0}}}=\frac{1}{2} \ln \frac{A}{A_{0}}=\frac{1}{2} \ln \left(\frac{l_{x}}{l_{x 0}} \frac{l_{z}}{l_{z 0}}\right)=\frac{1}{2}\left(\varepsilon_{x}+\varepsilon_{z}\right) \\
\bar{v}=-\frac{\Delta \bar{\varepsilon}_{\text {lateral }}}{\Delta \varepsilon_{y}}=-\frac{1 / 2\left(\Delta \varepsilon_{x}+\Delta \varepsilon_{z}\right)}{\Delta \varepsilon_{y}}=\frac{1}{2}\left(v_{x}+v_{z}\right) .
\end{gathered}
$$

As mentioned earlier, the lateral strain $\varepsilon_{x}$ (width direction) is higher at the treated surface than in the center part, while the lateral strain $\varepsilon_{z}$ (thickness direction) shows the opposite trend. These observations make us wonder whether the equivalent lateral strain $\bar{\varepsilon}_{\text {lateral }}$ has gradient along the depth. Based on Eq. [1], the evolution of $\Delta \bar{\varepsilon}_{\text {lateral }}=\bar{\varepsilon}_{\text {various depths }}-\bar{\varepsilon}_{\text {centeral }}$ at the various depths as a function of applied tensile strain for the gradient-structured sample are displayed in Figure 6, which shows that the equivalent lateral strain also has significant gradient along the depth. Moreover, this gradient for the equivalent lateral strain also starts from the beginning of the tensile deformation, and increases with increasing applied tensile strain, especially at the areas close the treated surface.

It is well known that the apparent Poisson's ratio remains essentially constant at the elastic range, and then gradually increases at the elasto-plastic transition stage, finally approaches, but seldom actually reaches, the values of $1 / 2$ (which is tantamount to the assumption of constant volume). ${ }^{[41]}$ Variation of the apparent Poisson's ratio with increasing applied tensile strain affects the evolution of cross-sectional area, and this in turn has direct influence on plastic tensile instability. For "perfect" homogeneous materials, the evolution of the apparent Poisson's ratio during the elasto-plastic transition can be described as ${ }^{[41]}$

$$
v=\frac{1}{2}-\left(\frac{1}{2}-v_{\mathrm{e}}\right)\left(\frac{E_{\mathrm{S}}}{E}\right),
$$

where $v_{\mathrm{e}}$ is the Poisson's ratio for elastic stage, $E$ is the elastic modulus, and $E_{\mathrm{S}}$ is the tangent modulus at the related strain, i.e., the slope of the stress-strain curve.

In order to better understand the deformation physics of the gradient structure, especially at the elasto-plastic transition stage, the evolution of $v_{x}$ and $v_{z}$ at various depths for the gradient-structured sample and the data for the untreated homogeneous $\mathrm{CG}$ structure are provided in Figures 7(a) and (b), respectively. As shown, there is a strong anisotropy for the two lateral apparent Poisson's ratios. In other words, $v_{x}$ and $v_{z}$ evolves very differently with increasing applied strain.

Disregarding the anisotropy, the equivalent apparent Poisson's ratio $\left(\bar{v}=\frac{1}{2}\left(v_{x}+v_{z}\right)\right)$ at various depths for the gradient-structured sample and for the untreated homogeneous CG sample is displayed in Figure 7(c), which shows a strong gradient along the depth for the equivalent Poisson's ratio in the gradient-structured sample. This indicates that the strain gradient occurred during the entire tensile loading, which is consistent with our earlier report. ${ }^{[21]}$ At the plastic deformation stage, 


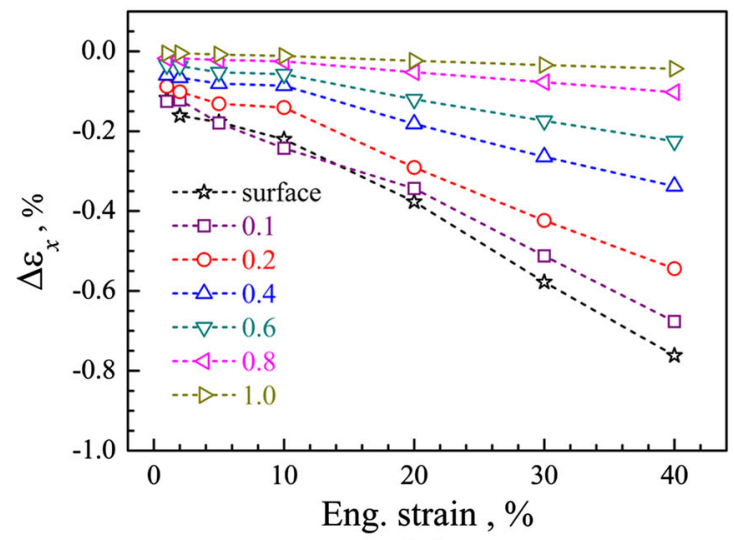

(a)

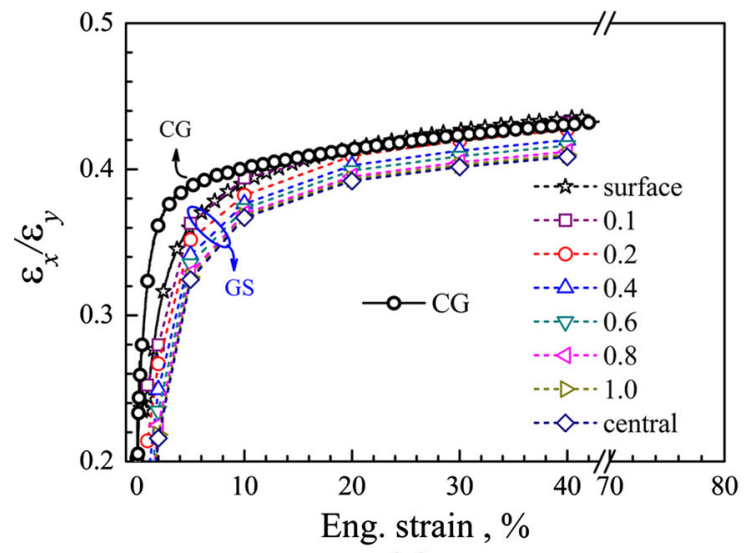

(c)

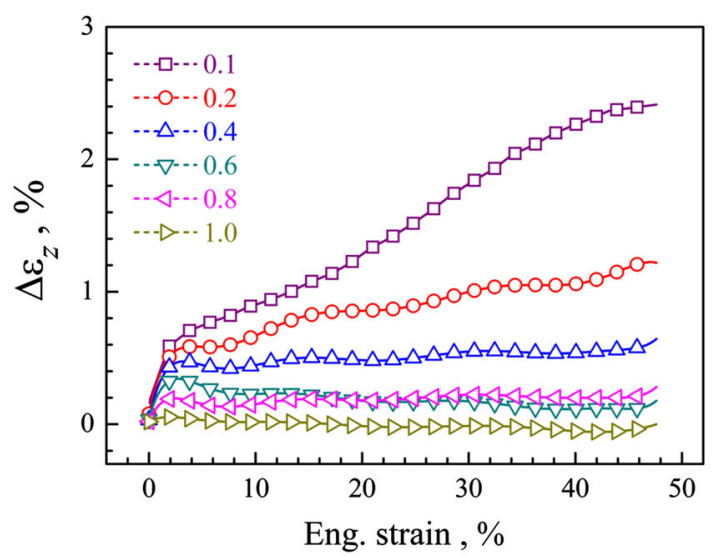

(b)

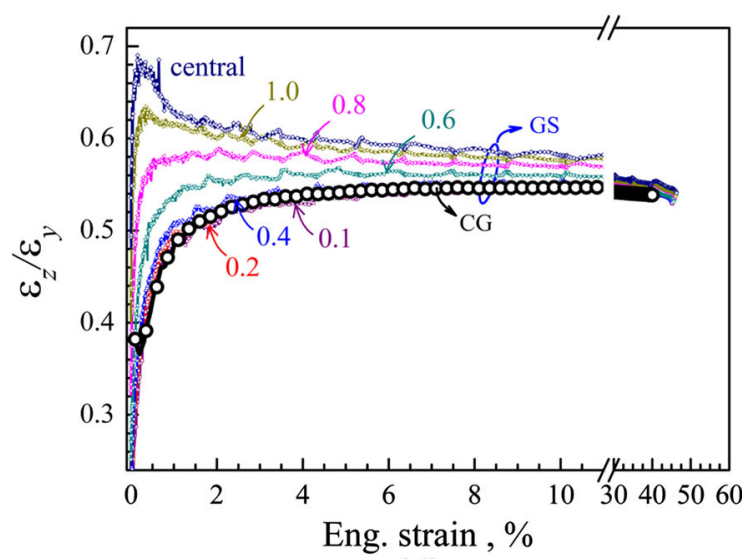

(d)

Fig. 5- (a) $\Delta \varepsilon_{x}$, the difference of $\varepsilon_{x}$ at the various depths and $\varepsilon_{x}$ at the central part as a function of applied tensile strain for the gradient-structured sample; (b) $\Delta \varepsilon_{z}$, the difference of $\varepsilon_{z}$ at the various depths and $\varepsilon_{x}$ at the central part as a function of applied tensile strain for the gradient-structured sample; $(c) \varepsilon_{x} / \varepsilon_{y}$ as a function of applied tensile strain at various depths for the gradient-structured sample; $(d) \varepsilon_{z} / \varepsilon_{y}$ as a function of applied tensile strain at various depths for the gradient-structured sample.

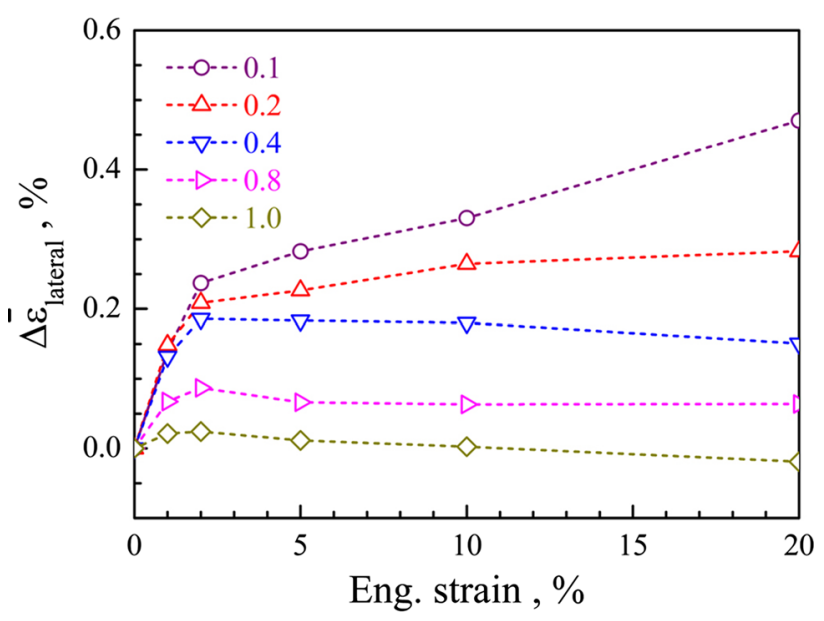

Fig. 6-The evolution of $\Delta \bar{\varepsilon}_{\text {lateral }}=\bar{\varepsilon}_{\text {various depths }}-\bar{\varepsilon}_{\text {centeral }}$ at various depths as a function of applied tensile strain for the gradient structure.

the equivalent apparent Poisson's ratio of the gradient-structured sample is slightly higher than those of the untreated homogeneous CG sample. This indicates that the reduction of the cross-section area is faster in the gradient structure than the untreated homogeneous $\mathrm{CG}$ structure at the plastic deformation stage. This observation is in good agreement with the fact that the strain hardening is weaker and the necking instability tendency is higher in the gradient-structured sample than in the untreated homogeneous CG sample.

In order to reveal the accumulated gradient for the equivalent lateral strain and its evolution at the small strain range, the normalized equivalent lateral strain $\left(\bar{\varepsilon}_{\text {lateral }} / \varepsilon_{y}\right)$ at various depths as a function of applied tensile strain is shown in Figure $7(\mathrm{~d})$. It is interesting to see from Figures 7(c) and (d) that the duration of the elasto-plastic transition stage is short for the homogeneous CG sample, which agrees well with the theoretical prediction. ${ }^{[41]}$ However, the duration of elasto-plastic transition stage is much longer in the gradient structure. In our previous work, ${ }^{[35]}$ a dual-phase steel with heterogeneous structure also exhibited a long and explicit elasto-plastic transition, which is mainly attributed to the load and strain partitioning between the two phases. The observed extraordinary strain-hardening rate in the dual-phase steel has been attributed to the high back-stress hardening from the strain 


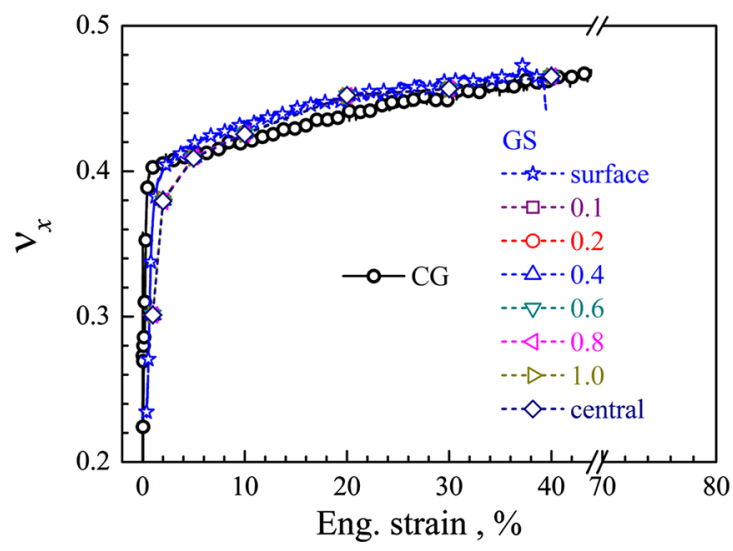

(a)

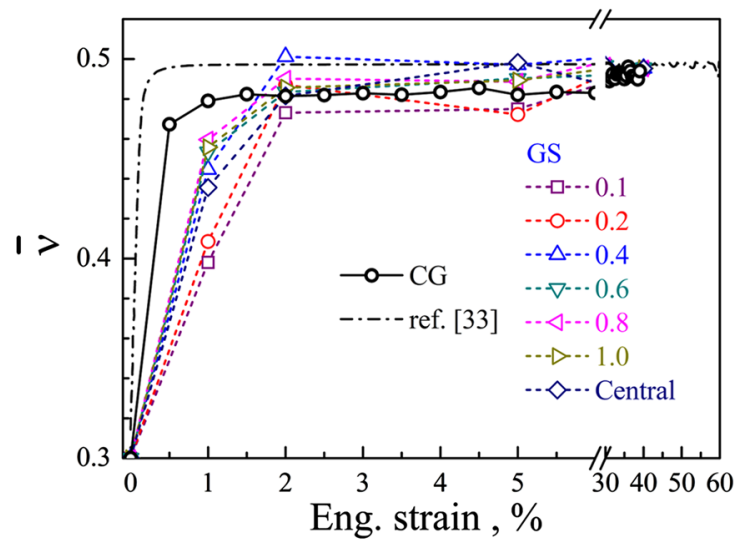

(c)

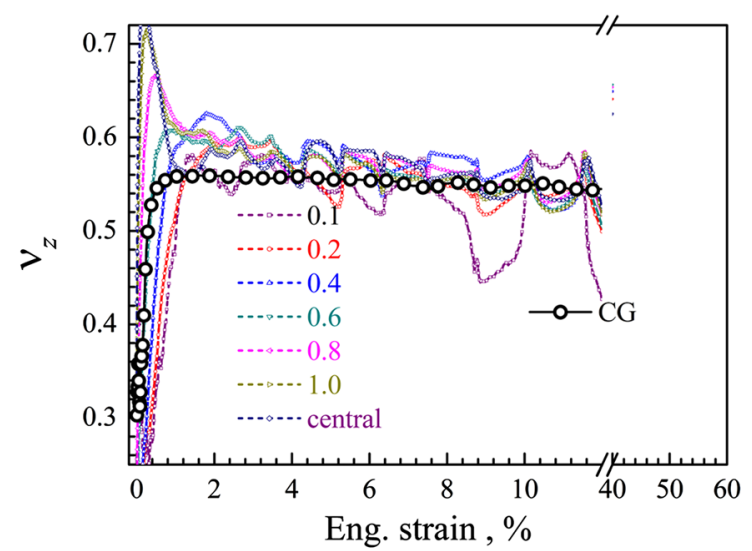

(b)

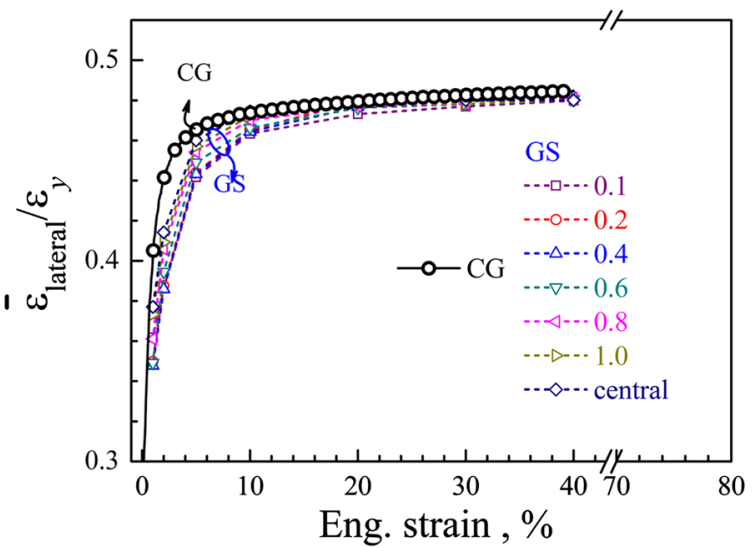

(d)

Fig. 7-(a) $v_{x}$ as a function of applied tensile strain at various depths for the gradient-structured sample; $(b) v_{z}$ as a function of applied tensile strain at various depths for the gradient-structured sample; (c) The equivalent Poisson's ratio $\left(\bar{v}=\frac{1}{2}\left(v_{x}+v_{z}\right)\right)$ as a function of applied tensile strain at various depths for the gradient-structured sample; $(d) \bar{\varepsilon}_{\text {lateral }} / \varepsilon_{y}$ as a function of applied tensile strain at various depths for the gradient-structured sample. The theoretical prediction from $v=\frac{1}{2}-\left(\frac{1}{2}-v_{e}\right)\left(\frac{E_{S}}{E}\right)$ for the homogeneous CG structure is also provided in (c) for comparison.

incompatibility between the two phases, which produced strain gradient near the phase boundaries. ${ }^{[35]}$ In the gradient structure, strain gradient also exists between different layers, and thus the mechanical incompatibility resulted in longer duration of elasto-plastic transition, providing strong back-stress hardening particularly at this stage. ${ }^{[24]}$

As mentioned earlier, deformation anisotropy in the two lateral directions is induced during the tensile loading in the gradient structure. In order to clearly illustrate this, $\varepsilon_{x}-\varepsilon_{z}$ curves as a function of applied tensile strain at various depths are presented as the accumulated deformation anisotropy in Figure 8(a), while $v_{z}-v_{x}$ curves are displayed as the instantaneous deformation anisotropy in Figure 8(b). For comparison, the curves for the homogeneous CG sample are also provided in Figure 8. As shown, the deformation anisotropy is higher in the gradient structure than in the homogeneous CG structure from the beginning of the tensile deformation and lasts all the way to the large applied tensile strain. The instantaneous deformation anisotropy is found to decrease, while the accumulated deformation anisotropy is observed to increase with increasing applied tensile strain. The high anisotropy in lateral deformation could intensify the interaction and the constraint between different layers at various depths, resulting in higher lateral stresses to promote the operation of additional slip systems and consequently leads to more efficient dislocation storage. ${ }^{[21]}$

\section{Back-Stress Hardening}

The gradient structures can be considered consisting of numerous thin layers with varying flow behaviors, which produces mechanical incompatibilities between neighboring layers. This in turn produces back stress, which is a long-range stress field that hinder dislocation slip to accommodate the applied strain. As a result, higher flow stress is needed to overcome this stress field and back-stress hardening is induced during the plastic deformation. This back-stress hardening mechanism have been observed in our recent works on a Ti lamella structure, ${ }^{[19]}$ an IF steel gradient structure, ${ }^{[24]}$ and a copper/bronze laminate structure. ${ }^{[28]}$ Figure 9 (a) shows the true stress-strain curves for LUR tests on both the CG sample and the gradient-structured sample, and the 


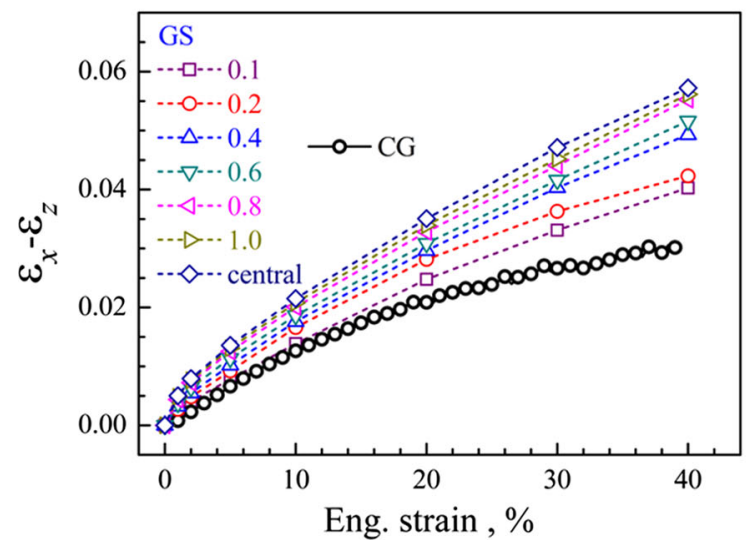

(a)

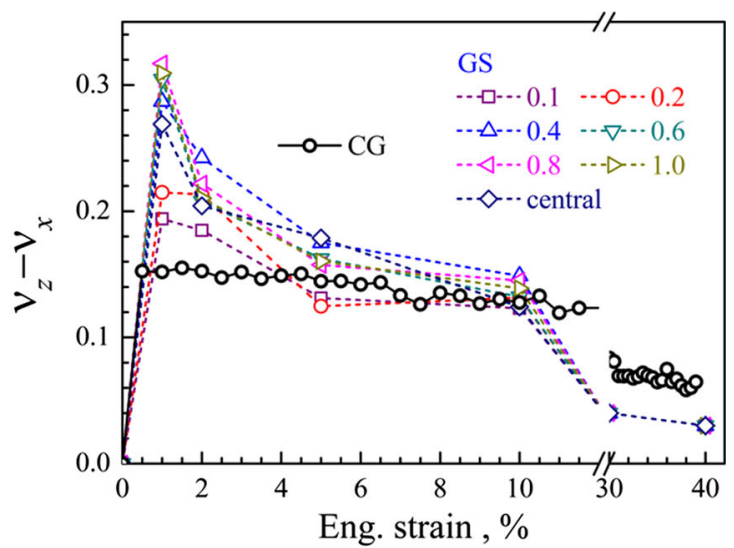

(b)

Fig. 8-(a) $\varepsilon_{x}-\varepsilon_{z}$ and $(b) v_{z}-v_{x}$ as a function of applied tensile strain at various depths for the gradient-structured and CG samples.

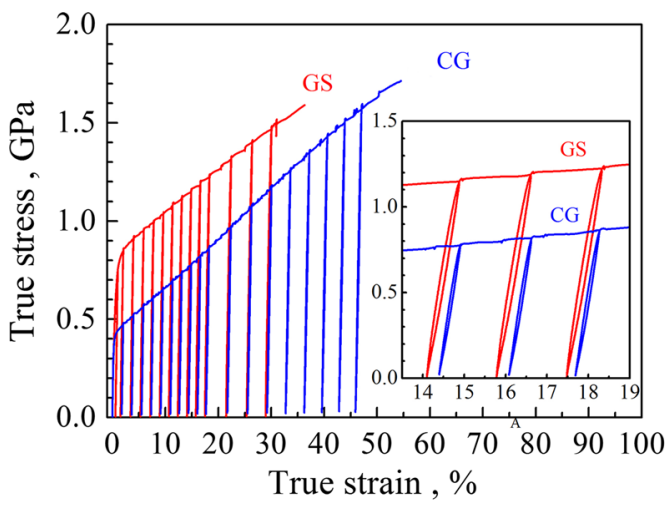

(a)

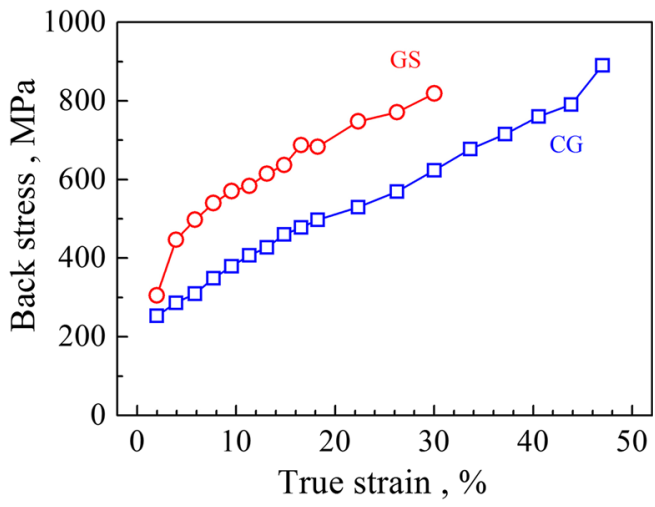

(b)

Fig. 9- (a) True stress-strain curves of LUR tests for the CG structure and the gradient structure; $(b)$ The calculated back stress at corresponding tensile strain levels.

inset displays the close-up view on the details of the hysteresis loops. The back stress is the stress that drives the mobile dislocations to move reversely to produce unloading yield ( $\sigma_{\mathrm{u}}$ is the unloading yield stress) during the unloading process $\left(\sigma_{\mathrm{b}}=\sigma_{\mathrm{u}}+\sigma_{\mathrm{f}}\right)$. During the reloading process, the applied stress needs to overcome the frictional stress $\left(\sigma_{\mathrm{f}}\right)$ and the back stress to drive the dislocation forward at the reloading yield point $\left(\sigma_{\mathrm{r}}\right.$ is the reloading yield stress, $\left.\sigma_{\mathrm{r}}=\sigma_{\mathrm{b}}+\sigma_{\mathrm{f}}\right)$. Thus, the back stress can be estimated by the average of the unloading yield stress and the reloading yield stress $\left(\sigma_{\mathrm{b}}=\left(\sigma_{\mathrm{u}}+\right.\right.$ $\left.\left.\sigma_{\mathrm{r}}\right) / 2\right) .{ }^{[24]}$ The back-stress evolution along with tensile loading (see Figure 9(b)) can be calculated from the unloading-reloading curves at varying tensile strains using an equation proposed in our recent work. ${ }^{[24]}$ It is found that the resulted back stress is much higher in the gradient structure than that in the CG structure, and the back-stress hardening is more pronounced in the gradient structure, especially at the early stage of tensile deformation. This high back-stress hardening rate at the early stage of tensile deformation for the gradient structure can be attributed to the elasto-plastic transition at this stage, at which the mechanical incompatibility is largest since the hard surface layers are still under elastic deformation, while the soft center layer is already under plastic deformation. In the co-deformation stage, all layers are under plastic deformation and back-stress hardening rate should become smaller for the gradient structure.

It is proposed in the previous work ${ }^{[42,43]}$ that the back-stress hardening in the CG structure can be understood by the development of internal stresses between the newly formed "hard" nanotwin lamellae and the "soft" untwined matrix during the tensile loading. The mechanical incompatibility between different layers with varying flow behaviors in the gradient structure also contributes significantly to the back-stress hardening, besides the back-stress hardening originated from the interaction between the "soft" matrix and newly formed "hard" twins in the CG grains at the center of the gradient structure. Consequently, more GNDs accumulate at the interfaces of different layers, resulting in a higher observed back stress and a more pronounced back-stress hardening in the gradient structure. Based on the current findings, the density of these GNDs due to the strain gradients increased with increasing applied strain to delay necking and to increase the ductility for the gradient-structured sample. 


\section{CONCLUDING REMARKS}

For the first time, the evolution of the strain gradient and deformation anisotropy in a gradient-structured sample during tensile loading were revealed by a series of quasi-static tensile tests, coupled with in-situ 3D strain contour measurements. The main findings are summarized below:

1. Unlike the homogeneous deformation in the untreated CG sample, strain gradients in both lateral directions started to develop at the beginning of the tensile deformation and increase with increasing applied tensile strain, resulting in increasing GNDs to sustain the uniform deformation for the gradient-structured sample.

2. Deformation anisotropy is much higher in the gradient structure than in the homogeneous CG structure. The accumulated deformation anisotropy is observed to increase with increasing applied tensile strain. This could intensify the interaction and the constraint between different layers at various depths, resulting in higher lateral stresses to promote the operation of additional slip systems to help with dislocation storage.

3. More pronounced back-stress hardening is observed in the gradient structure than in the CG structure, due to the plastic incompatibility between different layers with varying mechanical behaviors in the gradient structure. The high back-stress hardening can suppress necking to improve ductility in the gradient-structured sample.

\section{ACKNOWLEDGMENTS}

This work was financially supported by NSFC under Grant Nos. 11472286, 11572328, and 11672313, the 973 Program of China under Grant Nos. 2012CB932203 and 2012CB937500, and the Strategic Priority Research Program of the Chinese Academy of Sciences under Grant No. XDB22040503. Y.Z. was supported by the US Army Research Office (W911 NF-12-1-0009), the US National Science Foundation (DMT-1104667), and the Nanjing University of Science and Technology. This paper is dedicated to Professor Koch's TMS symposium 2017(Mechanical Behavior of Advanced Materials). This symposium will honor the outstanding contributions of Professor Carl C. Koch to many fields in materials science in the last 50 years and celebrate his 80th birthday.

\section{REFERENCES}

1. Y.T. Zhu and X.Z. Liao: Nat. Mater., 2004, vol. 3, pp. 351-52.

2. Y.M. Wang, M.W. Chen, F.H. Zhou, and E. Ma: Nature, 2002, vol. 419, pp. 912-15.

3. YH Zhao, XZ Liao, S Cheng, E Ma, and YT Zhu: Adv. Mater., 2006, vol. 18, pp. 2280-83.

4. K. Lu, L. Lu, and S. Suresh: Science, 2009, vol. 324, pp. 349-52.
5. K. Lu: Science, 2014, vol. 345, pp. 1455-56.

6. R.Z. Valiev: Nat. Mater., 2004, vol. 3, pp. 511-16.

7. M.A. Meyers, A. Mishra, and D.J. Benson: Prog. Mater. Sci., 2006, vol. 51, pp. 427-556.

8. M. Suzuki, T. Kimura, J. Koike, and K. Maruyama: Scripta Mater., 2003, vol. 48, pp. 997-1002.

9. S. Cheng, Y.H. Zhao, Y.T. Zhu, and E. Ma: Acta Mater., 2007, vol. 55, pp. 5822-32.

10. J. da Costa Teixeira, L. Bourgeois, C.W. Sinclair, and C.R. Hutchinson: Acta Mater., 2009, vol. 57, pp. 6075-89.

11. Y.T. Zhu, X.Z. Liao, and X.L. Wu: Prog. Mater. Sci., 2012, vol. 57, pp. 1-62.

12. R.Z. Valiev, Y. Estrin, Z. Horita, T.G. Langdon, M.J. Zehetbauer, and Y.T. Zhu: JOM, 2016, vol. 68, pp. 1216-26.

13. K.M. Youssef, R.O. Scattergood, K.L. Murty, J.A. Horton, and C.C. Koch: Appl. Phys. Lett., 2005, vol. 87, pp. 54-421.

14. L. Lu, X. Chen, X. Huang, and K. Lu: Science, 2009, vol. 323, pp. 607-10.

15. X.Y. Li, Y.J. Wei, L. Lu, K. Lu, and H.J. Gao: Nature, 2010, vol. 464, pp. 877-80.

16. L.L. Zhu, S.X. Qu, X. Guo, and J. Lu: J Mech. Phys. Solids., 2015, vol. 76, pp. 162-79.

17. P.V. Liddicoat, X.Z. Liao, Y.H. Zhao, Y.T. Zhu, M.Y. Murashkin, E.J. Lavernia, R.Z. Valiev, and S.P. Ringer: Nat. Commun., 2010, vol. 1, p. 63.

18. G. Liu, G.J. Zhang, F. Jiang, X.D. Ding, Y.J. Sun, J. Sun, and E. Ma: Nat. Mater., 2013, vol. 12, pp. 344-50.

19. X.L. Wu, M.X. Yang, F.P. Yuan, G.L. Wu, Y.J. Wei, X.X. Huang, and Y.T. Zhu: Proc. Natl. Acad. Sci. USA, 2015, vol. 112, pp. 14501-05.

20. T.H. Fang, W.L. Li, N.R. Tao, and K. Lu: Science, 2011, vol. 331, pp. 1587-90.

21. X.L. Wu, P. Jiang, L. Chen, F.P. Yuan, and Y.T. Zhu: Proc. Natl. Acad. Sci. USA, 2014, vol. 111, pp. 7197-201.

22. S Suresh: Science, 2001, vol. 292, pp. 2447-51.

23. Y.J. Wei, Y. Li, L. Zhu, Y. Liu, X. Lei, G. Wang, Y. Wu, Z. Mi, J. Liu, H. Wang, and H.J. Gao: Nat. Commun., 2014, vol. 5, p. 3580.

24. M.X. Yang, Y. Pan, F.P. Yuan, Y.T. Zhu, and X.L. Wu: Mater. Res. Lett., 2016, vol. 4, pp. 141-51.

25. Z. Zeng, X.Y. Li, D.S. Xu, L. Lu, H.J. Gao, and T. Zhu: Extreme Mech. Lett., 2016, vol. 8, pp. 213-19.

26. X.L. Wu, M.X. Yang, F.P. Yuan, L. Chen, and Y.T. Zhu: Acta Mater., 2016, vol. 112, pp. 337-46.

27. X.L. Ma, C.X. Huang, W.Z. Xu, H. Zhou, X.L. Wu, and Y.T. Zhu: Scripta Mater., 2015, vol. 103, pp. 57-60.

28. X.L. Ma, C.X. Huang, J. Moering, M. Ruppert, H.W. Höppel, M. Göken, J. Narayan, and Y.T. Zhu: Acta Mater., 2016, vol. 116, pp. 43-52.

29. R. Yuan, I.J. Beyerlein, and C.Z. Zhou: Mater. Res. Lett., 2016, DOI:10.1080/21663831.2016.1255264.

30. X.L. Wu, P. Jiang, L. Chen, and Y.T. Zhu: Mater. Res. Lett., 2014, vol. 2, pp. 185-91.

31. Y. Xiang and J.J. Vlassak: Acta Mater., 2006, vol. 54, pp. 5449-60.

32. J. Rajagopalan, J.H. Han, and M.T.A. Saif: Scripta Mater., 2008, vol. 59, pp. 734-37.

33. S.W. Lee, A.T. Jennings, and J.R. Greer: Acta Mater., 2013, vol. 61, pp. 1872-85.

34. X. Feaugas: Acta Mater., 1999, vol. 47, pp. 3617-32.

35. M.X. Yang, F.P. Yuan, Q.G. Xie, Y.D. Wang, E. Ma, and X.L. Wu: Acta Mater., 2016, vol. 109, pp. 213-22.

36. K. Lu and J. Lu: J. Mater. Sci. Technol., 1999, vol. 15, pp. 193-197.

37. A.G. Evans and J.W. Hutchinson: Acta Mater., 2009, vol. 57, pp. $1675-88$.

38. N.A. Fleck, G.M. Muller, M.F. Ashby, and J.W. Hutchinson: Acta Metall. Mater., 1994, vol. 42, pp. 475-87.

39. W.D. Nix and H.J. Gao: J. Mech. Phys. Solids, 1998, vol. 46, pp. 411-25.

40. M.F. Ashby: Philos. Mag., 1970, vol. 21, pp. 399-424

41. C.W. Bert, E.J. Mills, and W.S. Hyler: J. Basic Eng., 1967, vol. 89, pp. 35-39.

42. O. Bouaziz: Scripta Mater., 2012, vol. 66, pp. 982-85.

43. J.G. Sevillano and F.D.L. Cuevas: Scripta Mater., 2012, vol. 66, pp. $978-81$. 